\title{
CONCERNING THE CUT POINTS OF CONTINUA*
}

BY

GORDON T. WHYBURN

In this paper propositions will be established concerning the collection of all the cut points of a given plane $\dagger$ continuum. It will be shown that there does not exist an uncountable collection of mutually exclusive subcontinua of a given continuum $M$ each of which contains at least one cut point of $M$. With the aid of this fundamental theorem it is shown, among other things, that the set of all the cut points of a continuum $M$ which are irregular points of $M$ is necessarily countable and, indeed, that all save a countable number of the cut points of any continuum $M$ are points of Menger order two of $M$.

\section{Preliminary notions. Intervals and segments of a continuum}

Definitions. The points, or point sets, $A$ and $B$ of a continuum $M$ are said to be separated in $M$ by the point $X$ of $M$ provided that $M-X$ is the sum of two mutually separated point sets $M_{a}(X)$ and $M_{b}(X)$ containing $A$ and $B$ respectively. The notation $K(A, B)$ will be used to denote the set of all points of $M$ which separate $A$ and $B$ in $M$.

Let $A$ and $B$ be any two points of a continuum $M$ and $X$ and $Y$ any two distinct points of $K(A, B)$. Then $X$ will be said to precede $Y$ on $M$ in the order from $A$ to $B$ provided that $X$ belongs to the set $M_{a}(Y) ; X$ will be said to follow $Y$ provided that $X$ belongs to the set $M_{b}(Y)$. It is easily seen that if $X$ precedes $Y$, then $Y$ follows $X$. If $X$ precedes $Y$, then by the interval of $M$ from $X$ to $Y$ (or from $Y$ to $X$ ), or the interval $X Y$ (or $Y X$ ) of $M$, will be meant the set of points $\left[M_{b}(X)+X\right] \cdot\left[M_{a}(Y)+Y\right]$, and by the segment of $M$ from $X$ to $Y$ will be meant the set of points $M_{b}(X) \cdot M_{a}(Y)$. If $X$ follows $Y$, then since $Y$ precedes $X$, by the interval $X Y$ of $M$ is meant the set of points $\left[M_{b}(Y)+Y\right] \cdot\left[M_{a}(X)+X\right]$, and the segment $X Y$ (or $Y X$ ) of $M$ means the set of points $M_{a}(X) \cdot M_{b}(Y)$. The notation $I(X, Y)$ for the interval of $M$ from $X$ to $Y$ and $S(X, Y)$ for the segment of $M$ from $X$ to $Y$ will be used in this paper. Clearly $S(X, Y)=I(X, Y)-(X+Y)$.

The following two propositions can be proved with little difficulty from the above definitions.

* Presented to the Society, September 9, 1927; received by the editors in September, 1927.

$\dagger$ Quite recently I have established some very general results on the cuttings of continua, as a consequence of which it follows that all the theorems in this paper hold true in a euclidean space of $n$ dimensions, and nearly all of them hold in any separable metric space which is locally compact. See my forthcoming paper Concerning collections of cuttings of connected point sets. 
Theorem 1. Let $A$ and $B$ be any two points of a continuum $M$, and let $X$ and $Y$ be any two distinct points of $K(A, B)$, where $X$ precedes $Y$. Then $M$ is the sum of three continua $M_{a}(X)+X, I(X, Y)$, and $M_{b}(Y)+Y$, where $\left[M_{a}(X)+X\right] \cdot I(X, Y)=X,\left[M_{b}(Y)+Y\right] \cdot I(X, Y)=Y$, and $\left[M_{a}(X)+X\right]$ $\cdot\left[M_{b}(Y)+Y\right]=0$.

TheOREM 2. Using the same notation as in Theorem 1, suppose that each of the sets $M_{a}(X), M_{b}(X), M_{a}(Y)$, and $M_{b}(Y)$ is connected. Then if $S(X, Y)$ contains a point of $K(A, B), S(X, Y)$ is connected.

\section{THE PRINCIPAL RESULTS}

THEOREm 3. If $B$ is any uncountable subset of the set $G$ of all the cut points of a continuum $M$, then there exist two points $A$ and $B$ of $B$ which are separated in $M$ by uncountably many of the points of $H .{ }^{*}$

There exists a countable subset $D$ of $M$ such that every point of $M$ is a limit point of $D$. Let $F$ be the collection of all possible pairs of points of $D$. Then $F$ is countable. For each pair of points $u, v$ of $F$, let $K_{h}(u, v)$ denote the set of all points of $H$ that separate $u$ and $v$ in $M$. Then $H=\sum_{F} K_{h}(u, v)$. For let $X$ be a point of $H$. Then since $X$ is a cut point of $M, M-X=M_{1}$ $+M_{2}$, where $M_{1}$ and $M_{2}$ are mutually separated point sets. Since every point of $M$ is a limit point of $D$, it follows that $M_{1}$ contains a point $U$ of $D$ and $M_{2}$ contains a point $V$ of $D$. Then $X$ separates $U$ and $V$ in $M$, and hence it belongs to $K_{h}(U, V)$. Therefore $H=\sum_{F} K_{h}(u, v)$; and since $F$ is countable and $B$ is uncountable, it follows that there exists at least one pair $P, Q$ of $F$ such that $K_{h}(P, Q)$ is uncountable.

Let $E$ denote the set of points $K_{h}(P, Q)$. Then since $E$ is uncountable, it contains a point $A$ which is a point of condensation of $E$, i.e., every vicinity of $A$ contains uncountably many points of $E$. Now for at least one of the sets $M_{p}(A)$ and $M_{q}(A)$, say for $M_{q}(A)$, it is true that $A$ is a point of condensation of the subset of $E$ which belongs to $M_{q}(A)$. Let $B$ be a point of $E$ belonging to $M_{q}(A)$. Then by Theorem 1 it readily follows that the segment $A B$ of $M$ must contain an uncountable subset $E_{0}$ of $E$. Let $X$ be any point of $E_{0}$. Then since $X$ belongs to $S(A, B)$, it follows by Theorem 1 that neither of the continua $M_{p}(A)+A$ and $M_{q}(B)+B$ contains the point $X$. And as these continua contain the points $P$ and $Q$ respectively, therefore they are subsets of $M_{p}(X)$ and $M_{q}(X)$ respectively. Hence $A$ and $B$ are separated in $M$ by each point $X$ of $E_{\mathrm{c}}$, and as $E_{0}$ is an uncountable subset of $H$, the truth of Theorem 3 is therefore established.

* The referee has kindly called my attention to the fact that Theorems 3 and 4 of this paper are valid in any number of dimensions, since my proofs of these theorems make no use of the dimensionality of the space in which the point sets lie. 
ThEOREM 4. Let $G$ denote the set of all the cut points of a continuum $M$. Then for all, save possibly a countable number, of points $X$ of $G$ it is true that $M-X$ is the sum of two mutually separated and connected point sets.*

Suppose, on the contrary, that there exists an uncountable subset $D$ of $G$ such that if $X$ is any point of $D$, then $M-X=M_{1}(X)+M_{2}(X)$, where $M_{1}(X)$ and $M_{2}(X)$ are mutually separated point sets and at least one of them is not connected. Then by Theorem 3 there exist two points $A$ and $B$ of $D$ which are separated in $M$ by each point of an uncountable subset $H$ of the set $D$. For each point $X$ of $H, M-X=H_{a}(X)+H_{b}(X)+H_{0}(X)$, where $H_{a}(X), H_{b}(X)$, and $H_{0}(X)$ are mutually separated point sets, and $H_{a}(X)$ and $H_{b}(X)$ contain $A$ and $B$ respectively.

Now for each point $X$ of $H$, the set $H$, and indeed the set $K(A, B)$, is a subset of the set of points $H_{a}(X)+H_{b}(X)+X$, because this set of points is connected and contains both $A$ and $B$ and therefore must contain every point of $M$ which separates $A$ and $B$ in $M$. It follows that if $X$ and $Y$ are distinct points of $H$, the set of points $H_{0}(Y)$ belongs to the set $H_{a}(X)+H_{b}(X)$, and the set $H_{0}(X)$ belongs to the set $H_{a}(Y)+H_{b}(Y)$; and therefore the sets $H_{0}(X)$ and $H_{0}(Y)$ are mutually exclusive. By the Zermelo postulate there exists a set of points $E$ such that for each point $X$ of $H$ there is exactly one point $P_{x}$ of $E$ which belongs to the set of points $H_{0}(X)$, and for each point $P_{x}$ of $E$ there is exactly one point $X$ of $H$ such that the set $H_{0}(X)$ contains the point $P_{x}$. Since $H$ is uncountable, $E$ must be uncountable. Hence there exists a point $P_{z}$ of $E$ which is a limit point of $E$. But there exists a point $Z$ in $H$ such that $P_{z}$ belongs to the set $H_{0}(Z)$, and $E-P_{z}$ belongs to the set of points $H_{a}(Z)+H_{b}(Z)$. Clearly this is impossible, because $H_{a}(Z), H_{b}(Z)$, and $H_{0}(Z)$ are mutually separated point sets. Thus the supposition that Theorem 4 is not true leads to a contradiction.

Theorem 4 can also be proved very readily with the aid of a statement given, without proof, by C. Zarankiewicz in his paper Sur les points de division dans les ensembles connexes. $\dagger$

Theorem 5. Let $M$ be any continuum whatever. Then there does not exist an uncountable collection of mutually exclusive subcontinua of $M$ each of which contains at least one cut point of $M$.

\footnotetext{
* Since this paper was submitted to the editors, a result somewhat more general than Theorem 4 has been published by Kuratowski andZarankiewicz; cf. A theorem on connected point sets, Bulletin of the American Mathematical Society, vol. 33 (1927), pp. 571-575.

$\dagger$ Cf. Fundamenta Mathematicae, vol. 9, pp. 124-171, footnote to p. 137.

¥ In this paper the hypothesis that a set is a continuum is understood to imply that the set contains more than one point.
} 
Before proceeding with the proof of Theorem 5, I shall establish two lemmas which will be needed in the proof.

LEMMa 1. If $C$ is any subcontinuum of a continuum $M$, then not more than a. countable number of the bounded maximal connected subsets of $M-C$ can contain cut points of $M$.

Suppose, on the contrary, that there exists an uncountable collection $G$ of bounded maximal connected subsets of $M-C$ each of which contains at least one cut point of $M$. From each element $g$ of $G$ select exactly one cut point $P_{o}$ of $M$, and let $K$ denote the set of all points $\left[P_{\theta}\right]$ thus selected. For each point $P_{o}$ of $K, M-P_{o}=M_{c}\left(P_{\vartheta}\right)+M_{0}\left(P_{\vartheta}\right)$, where $M_{c}\left(P_{o}\right)$ and $M_{0}\left(P_{\vartheta}\right)$ are mutually separated sets and $M_{c}\left(P_{o}\right)$ contains the continuum $C$. Let $H$ denote the sum of all the point sets of the collection $G$. Then since each element of $G$ has* at least one limit point in $C$, it follows that for each point $P_{o}$ of $K$, the set of points $H-g$ belongs to the set $M_{c}\left(P_{g}\right)$. By the Zermelo postulate, there exists a set of points $E$ such that for each point $P_{\theta}$ of $K$ there is exactly one point $Q_{\sigma}$ of $E$ which belongs to the set of points $M_{0}\left(P_{o}\right)$, and for each point $Q_{\theta}$ of $E$ there is exactly one point $P_{\theta}$ of $K$ such that the set of points $M_{0}\left(P_{g}\right)$ contains the point $Q_{g}$. It is readily seen that no two of the sets $\left[M_{0}\left(P_{o}\right)\right]$ can have a common point. Therefore, as $H$ is uncountable, both $K$ and $E$ must be uncountable. Hence $E$ contains a point $Q_{j}$ which is a limit point of $E$. But clearly this is impossible, because there exists a point $P_{j}$ in $K$ such that $Q_{j}$ belongs to the set $M_{0}\left(P_{j}\right)$ and $E-Q_{j}$ belongs to the set $M_{c}\left(P_{j}\right)$, and $M_{0}\left(P_{j}\right)$ and $M_{i}\left(P_{i}\right)$ are mutually separated sets by definition. This contradiction proves Lemma 1.

Lemma 2. If $M$ is any continuum, $C$ is any simple closed curve, and $G_{0}$ denotes the collection of all the maximal connected subsets of $M$ contained in $C$ plus its interior, then not more than a countable number of elements of $G_{0}$ can contain cut points of $M$ which lie within $C$.

Suppose, on the contrary, that $G_{0}$ contains an uncountable subcollection $G$ each element $g$ of which contains at least one cut point $P_{\sigma}$ of $M$ lying within C.

(1) Not more than a countable number of elements $g$ of $G$ can have the property that $M-P_{\delta}$ can be expressed as the sum of two mutually separated sets one of which lies wholly within $C$. For suppose the contrary is true. Then, clearly, for each such element $g$ of $G$, the point $P_{\theta}$ is a cut point of the continuum $M+C$. But since each such point $P_{\theta}$ lies on a bounded maximal

* See my paper Concerning point sets which can be made connected by the addition of a simple continuous arc, these Transactions, vol. 29 (1927), footnote to p. 754. 
connected subset of $(M+C)-C$, and no two different points $\left[P_{o}\right]$ can lie on the same maximal connected subset of $(M+C)-C$, this is contradictory to Lemma 1.

(2) Let $G_{1}$ denote the collection of all those elements $g$ of $G$ such that however $M-P_{o}$ be expressed as the sum of two mutually separated point sets $M_{1}\left(P_{0}\right)$ and $M_{2}\left(P_{0}\right)$, each of these sets must contain at least one point of $C$. It follows by (1) that $G_{1}$ must be uncountable. It is clear that if $g$ is any element of $G_{1}$ and we let $g_{1}(P)=g \cdot M_{1}\left(P_{o}\right)$ and $g_{2}(P)=g \cdot M_{2}\left(P_{g}\right)$, then $g-P_{o}=g_{1}(P)+g_{2}(P)$, and $g_{1}(P)$ and $g_{2}(P)$ are mutually separated point sets each of which contains at least one point on $C$. Now $C$ is not a subset of $M$, for if it were, then $G_{0}$ would have only one element. Let the maximal segments of $C-M \cdot C$ be denoted by $S_{1}, S_{2}, S_{3}, \ldots$. Let $g$ be any element of $G_{1}$. Denote the end points of $S_{1}$ by $A$ and $B$, and let $A O B$ be the arc of $C$ from $A$ to $B$ which does not contain $S_{1}$. On $A O B$, in the order from $A$ to $B$, let $X_{\theta}$ be the first point belonging to $g$. Then $X_{\theta}$ belongs either to $g_{1}(P)$ or to $g_{2}(P)$, say to $g_{1}(P)$. Then on the $\operatorname{arc} X_{\theta} B$ of $A O B$, in the order from $X_{\sigma}$ to $B$, let $Y_{\theta}$ be the first point belonging to $g_{2}(P)$. It is easy to see that the segment $X_{v} Y_{v}$ of the arc $A O B$ contains at least one segment $S_{i}$ of the sequence $S_{1}, S_{2}, S_{3}, \cdots$. Let $A_{i}$ and $B_{i}$ denote the end points of $S_{i}$, where $A_{i}$ precedes $B_{i}$ on $A O B$ in the order from $A$ to $B$. Then if $T_{1}$ and $T_{2}$ denote the $\operatorname{arcs} A A_{i}$ and $B_{i} B$ respectively of $A O B, g_{1}(P)$ has at least one point on one of these arcs and $g_{2}(P)$ has at least one point on the other. Thus it is seen that for each element $g$ of $G_{1}$ there exist two segments of the sequence $S_{1}, S_{2}, S_{3}, \cdots$ such that $g_{1}(P)$ has a point on one of the complementary arcs of $C$ of these two segments and $g_{2}(P)$ has a point on the other. Then since the number of segments $S_{1}, S_{2}, S_{3}, \cdots$ is countable, and $G_{1}$ is uncountable, there exist two segments $S_{k}$ and $S_{j}$ of the sequence $S_{1}, S_{2}, S_{3}, \cdots$ such that if $T$ and $L$ denote the complementary $\operatorname{arcs}$ of $C$, then for uncountably many elements $g$ of $G_{1}$ it is true that $g_{1}(P)$ has at least one point on one of the $\operatorname{arcs} T$ and $L$, and $g_{2}(P)$ has at least one point on the other. Let $G_{2}$ denote the collection of all those elements of $G_{1}$ which have this property.

(3) There are not more than a countable number of elements $g$ of $G_{2}$ such that $T+L$ contains as many as three points of $g$. For suppose the contrary is true. Then there exists an uncountable subcollection $E$ of $G_{2}$ such that each element $e$ of $E$ has at least two points on one of the $\operatorname{arcs} T$ and $L$, say on $T$, and at least one point on $L$. Let $A$ and $B$ denote the end points of $T$, where $A$ is an end point of $S_{k}$, and for each element $e$ of $E$, let $A_{\bullet}$ and $B$. respectively denote the first and last points on $T$ in the order from $A$ to $B$ which belong to $e$. Then for no two elements $e$ and $f$ of $E$ can the segments 
$A_{e} B_{e}$ and $A_{f} B_{f}$ of $T$ have a common point. For suppose they do. Then either the segment $A_{e} B_{\mathrm{e}}$ contains a point of $f$ or the segment $A_{f} B_{f}$ contains a point of $e$. The two cases are alike, so let us suppose that the segment $A_{0} B$. contains a point $X$ of $f$. Now $L$ contains at least one point $Y$ of $f$. There exists an arc $X Z Y$ from $X$ to $Y$ which lies, except for the points $X$ and $Y$, wholly without $C$. It is readily seen that the segments $S_{k}$ and $S_{j}$ belong to different complementary domains $R_{k}$ and $R_{i}$ respectively of the continuum $f+X Z Y$. Since $A_{e}$ can be joined to $A$ and $B_{c}$ to $B$ by arcs lying, except for their end points, in the exterior of $C$ and containing no point whatever of $X Z Y$, it follows that $A_{e}$ must belong to $R_{k}$ and $B_{e}$ to $R_{j}$. Then since $e$ is connected, it must have at least one point in common with $f+X Z Y$. But as the segment $X Z Y$ lies without $C, e$ can have no point in common with it. Hence $e$ must have a point in common with $f$. This is contradictory to the fact that $e$ and $f$ are different maximal connected subsets of $M$ lying in $C$ plus its interior. Therefore no two segments $A_{e} B_{e}$ and $A_{f} B_{f}$ can have a common point. But since $E$ is uncountable, then the number of these segments must be uncountable. Clearly this is impossible. This contradiction proves the statement made in the first sentence of this paragraph.

(4) It follows from (3) that there exists an uncountable subcollection $G_{3}$ of elements of $G_{2}$ such that if $e$ is any element of $G_{3}$, then $e_{1}(P)$ has exactly one point $X_{0}$ on $T$ and $e_{2}(P)$ has exactly one point $Y_{\bullet}$ on $L$. But since $G_{3}$ is uncountable, it contains an element $g$ which contains the sequential limiting set $H$ of some infinite sequence $V$ of elements of $G_{3}$. Then $H$ is connected and contains at least one point on each of the $\operatorname{arcs} T$ and $L$. But since $g$ has in common with $T+L$ only the points $X_{\theta}$ and $Y_{\theta}, H$ must contain both $X_{\theta}$ and $Y_{g}$. Now some infinite subsequence $V_{1}$ of $V$ has the property that all of its elements belong to one of the sets $M_{1}\left(P_{\theta}\right)$ and $M_{2}\left(P_{\theta}\right)$, say to $M_{1}\left(P_{o}\right)$. But $H$ is also the limiting set of the sequence $V_{1}$, and the point $Y_{o}$ belongs to the set $M_{2}\left(P_{o}\right)$. Hence $M_{2}\left(P_{o}\right)$ contains a limit point of $M_{1}\left(P_{o}\right)$, contrary to the fact that $M_{1}\left(P_{o}\right)$ and $M_{2}\left(P_{o}\right)$ are mutually separated point sets. Thus the supposition that Lemma 2 is not true leads to a contradiction.

Proof of Theorem 5. Suppose, contrary to Theorem 5, that there exists an uncountable collection $G$ of mutually exclusive subcontinua of $M$ each of which contains at least one cut point of $M$. From each element $g$ of $G$ select exactly one cut point $P_{\sigma}$ of $M$, and let $N$ denote the set of all points $\left[P_{g}\right]$ thus selected. Since $N$ is uncountable, there exists a square $S$ which encloses uncountably many points of $N$. Let $N_{1}$ be the set of all those points of $N$ which are within $S$. There exists a positive number $d$ and an uncountable subset $N_{2}$ of $N_{1}$ such that if $G_{2}$ denotes the collection of all those elements 
of $G$ which contain a point of $N_{2}$, then each element of $G_{2}$ is of diameter greater than $4 d$. Let us divide $S$ plus its interior into a finite collection $E$ of squares plus their interiors by lines parallel and perpendicular to the bases of $S$ in such a way that the diameter of each square of $E$ is less than $d$. Then either some side $T$ of one of the squares of $E$ contains uncountably many points of $N_{2}$, or else some square of $E$ encloses uncountably many of these points. In either case it is readily seen that there exists a rectangle $R_{1}$ of diameter less than $2 d$ which encloses uncountably many of the points of $N_{2}$. There exists a rectangle $R_{2}$ concentric with and within $R_{1}$ which also encloses an uncountable subset $N_{3}$ of $N_{2}$.

Let $F$ denote the collection of all the maximal connected subsets of $M$ contained in $R_{2}$ plus its interior. Since $N_{3}$ is uncountable and each point of $N_{3}$ belongs to some element of $F$, and since, by Lemma 2, not more than a countable number of elements of $F$ can contain points of $N_{3}$, it follows that some element $K$ of $F$ must contain an uncountable subset $N_{4}$ of $N_{3}$. It follows by a theorem of R. L. Moore's* that uncountably many points of $N_{4}$ must be cut points also of the continuum $K$. Let $N_{5}$ be the set of all those points of $N_{4}$ which are cut points of $K$. By Theorem 3, there exist two points $A$ and $B$ of $N_{5}$ which are separated in $K$ and also in $M$ by uncountably many points of $N_{5}$. Let $N_{6}$ be the set of all those points of $N_{5}$ which separate $A$ and $B$ in $K$ and also in $M$, and let $G_{6}$ be the collection of all those elements of $G_{2}$ which contain a point of $N_{6}$. Since each element of $G_{6}$ is of diameter greater than $4 d$, and $R_{1}$ is of diameter less than $2 d$, each element of $G_{6}$ must contain a point of $R_{1}$ and also a point of $R_{2}$. For each element $g$ of $G_{6}$, the maximal connected subset $H_{0 a}$ of $g$ which contains the point $P_{0}$ and lies within $R_{1}$ has at least one limit point $A_{P_{g}}$ which belongs to $R_{1}$. Let $H_{P_{g}}$ denote the continuum obtained by adding to $H_{0 \sigma}$ all of its limit points. Now since $A$ and $B$ belong to $N_{5}$, the elements $g_{a}$ and $g_{b}$ of $G_{2}$ which contain $A$ and $B$ respectively contain subcontinua $H_{a}$ and $H_{b}$ respectively which contain $A$ and $B$ respectively and at least one point $U$ and $V$ respectively on $R_{1}$ but which lie wholly in $R_{1}$ plus its interior. It follows by Theorem 4 that $N_{6}$ contains an uncountable subset $N_{7}$ such that if $X$ is any point of $N_{7}$, then $M-X=M_{a}(X)+M_{b}(X)$, where $M_{a}(X)$ and $M_{b}(X)$ are mutually separated and connected point sets containing $A$ and $B$ respectively, and also $K-X=K_{a}(X)+K_{b}(X)$, where $K_{a}(X)$ and $K_{b}(X)$ are connected sets belonging to $M_{a}(X)$ and $M_{b}(X)$ respectively. Let $E_{0}$ denote the collection of all the continua $\left[H_{x}\right]$ selected above which correspond to points $X$ of $N_{7}$. At least one of the arcs of $R_{1}$ from $U$ to $V$ must contain uncountably many points

* R. L. Moore, Concerning the cut points of continuous curves and of other closed and connected point sets, Proceedings of the National Academy of Sciences, vol. 9 (1923), pp. 101-106. 
$A_{x}$ which correspond to points $X$ of $N_{7}$ and belong to continua $H_{x}$ of the collection $E_{0}$; let $U O V$ denote one which does, and let $N_{8}$ be the set of all those points of $N_{7}$ such that the corresponding point $A_{x}$ belongs to the arc UOV.

Now if $X$ and $Y$ are two points of $N_{8}$ such that $X$ precedes $Y$ on $K$ in the order from $A$ to $B$ (see $\$ 1$ ), I shall show that the point $A_{x}$ precedes the point $A_{\nu}$ on the arc $U O V$ in the order from $U$ to $V$. Suppose, on the contrary, that $A_{y}$ precedes $A_{x}$ on $U O V$ in the order from $U$ to $V$. Then $U$ and $A_{x}$ separate the points $V$ and $A_{y}$ on $R_{1}$, and if $U W A_{x}$ is an arc from $U$ to $A_{x}$ which lies, except for its end points, in the exterior of $R_{1}$, then it is easy to see that the points $A_{y}$ and $V$ lie in different complementary domains of the continuum $U W A_{x}+H_{a}+K_{a}(X)+H_{x}$. Then since the continuum $H_{b}+K_{b}(Y)$ $+H_{y}$ contains both $A_{y}$ and $V$, therefore it must contain at least one point in common with $U W A_{x}+H_{a}+K_{a}(X)+H_{x}$. This is impossible, for no one of the sets $H_{b}, K_{b}(Y)$, and $H_{y}$ can have a point in common with any one of the sets $U W A_{x}, H_{a}, K_{a}(X)$, and $H_{x}$. Thus the supposition that $A_{x}$ does not precede $A_{y}$ on the arc $U O V$ in the order from $U$ to $V$ leads to a contradiction.

Now since $N_{8}$ is uncountable, the set of corresponding points $\left[A_{x}\right]$ is uncountable. Accordingly there exists one of these points $A_{z}$ which is a limit point of a subset $S_{1}$ of the remaining ones belonging to the arc $U A_{z}$ of $U O V$ and also of a subset $S_{2}$ of the remaining ones belonging to the arc $A_{z} V$ of $U O V$. Now the point $A_{z}$ belongs either to $M_{a}(Z)$ or to $M_{b}(Z)$, say to $M_{a}(Z)$. But this is impossible, since $A_{z}$ is a limit point of $S_{2}$, and if $A_{x}$ is any point of $S_{2}$, it was shown above that the point $X$ follows the point $Z$ on $M$ in the order from $A$ to $B$ (for the order on $M$ and on $K$ is the same), i.e., $X$ belongs to $M_{b}(Z)$; and thus $A_{z}$ is a limit point of $M_{b}(Z)$, which is absurd. Likewise we arrive at a contradiction if we suppose that $A_{z}$ belongs to $M_{b}(Z)$. Thus the supposition that Theorem 5 is not true leads to a contradiction and the theorem is therefore established.

THEOREM 6. If $E$ is any subset of the set of all the cut points of a continuum $M$, then there are not more than a countable number of points $X$ of $E$ such that $X$ belongs to some subcontinuum of $M$ which contains no other point of $E$.

Suppose, on the contrary, that there exists an uncountable subset $D$ of $E$ such that each point $X$ of $D$ belongs to some subcontinuum $N_{x}$ of $M$ containing no other point of $E$. Then by Theorems 3 and 4 it follows that there exist two points $A$ and $B$ of $M$ and an uncountable subset $F$ of $D$ such that if $X$ is any point of $F, X$ separates $A$ and $B$ in $M$ and furthermore each of the sets $M_{a}(X)$ and $M_{b}(X)$ is connected. 
Now for not more than a countable number of points $X$ of $F$ is it true that there exists some other point $Y$ of $F$ such that the segment $X Y$ of $M$ contains no point whatever of $F$. For let $Q$ be the set of all points $X$ of $F$ such that there exists a point $Y$ of $F$ such that $S(X, Y)$ contains no point of $F$, and suppose, contrary to the stacement just made, that $Q$ is uncountable. It follows that there exists an uncountable collection of the segments $[S(X, Y)]$ no two of which are identical and no one of which contains a point of $F$. Let $S\left(X_{1}, Y_{1}\right)$ and $S\left(X_{2}, Y_{2}\right)$ be any two of these segments, where $X_{1}$ precedes $Y_{1}$ and $X_{2}$ precedes $Y_{2}$ on $M$ in the order from $A$ to $B$. Then $S\left(X_{1}, Y_{1}\right)$ and $S\left(X_{2}, Y_{2}\right)$ are mutually exclusive. For suppose they have a point $P$ in common. One of these segments is not a subset of the other one, for they are not identical. Suppose $S\left(X_{2}, Y_{2}\right)$ is not a subset of $S\left(X_{1}, Y_{1}\right)$. Let $K$ denote the set of points common to $S\left(X_{1}, Y_{1}\right)$ and $S\left(X_{2}, Y_{2}\right)$. Now not both of the points $X_{1}$ and $Y_{1}$ can be limit points of $K$; for if they were, they would both belong to $I\left(X_{2}, Y_{2}\right)$ and at least one of them would have to belong to $S\left(X_{2}\right.$, $\left.Y_{2}\right)$, contrary to the fact that $S\left(X_{2}, Y_{2}\right)$ contains no point of $F$. The two cases are alike, so let us suppose $X_{1}$ is not a limit point of $K$. Then it is easy to see that $K$ is closed except possibly for the point $Y_{1}$. Since $K$ belongs to $S\left(X_{1}\right.$, $\left.Y_{1}\right)$, it must belong to $M_{a}\left(Y_{1}\right)$. But $M_{a}\left(Y_{1}\right)$ is connected; and since $M_{a}\left(Y_{1}\right)-K$ contains no limit point of $K$, therefore $K$ must contain at least one limit point of $M_{a}\left(Y_{1}\right)-K$. But clearly this is impossible, since by Theorem 1 it follows that $S\left(X_{1}, Y_{1}\right)$ and $S\left(X_{2}, Y_{2}\right)$ are open subsets of $M$. Thus the supposition that $S\left(X_{1}, Y_{1}\right)$ and $S\left(X_{2}, Y_{2}\right)$ are not mutually exclusive leads to a contradiction. Hence no two of the segments of the collection $[S(X, Y)]$ can have a common point. But since, by Theorem 1, each of these segments is an open subset of $M$ and since $[S(X, Y)]$ is an uncountable collection, this clearly is impossible. Thus the supposition that $Q$ is uncountable leads to a contradiction.

Now let $H$ denote the set of points $F-Q$. Then since $F$ is uncountable and $Q$ is countable, $H$ must be uncountable. By hypothesis, for each point $X$ of $H$ there exists a subcontinuum $N_{x}$ of $M$ which contains $X$ but which contains no other point whatever of $E$. Now if $X$ and $Y$ are any two distinct points of $B$, the continua $N_{x}$ and $N_{\nu}$ can have no point in common. For suppose they do have a point $P$ in common. The point $Y$ belongs either to $M_{a}(X)$ or to $M_{b}(X)$, say to $M_{b}(X)$. Since $X$ and $Y$ belong to $H$, the segment $X Y$ of $M$ must contain at least one point $O$ of $F$, and $O$ separates $A$ and $B$ in $M$. But the set of points $M_{a}(X)+M_{b}(Y)+N_{x}+N_{y}$ is connected and contains both $A$ and $B$ and does not contain the point $O$. Clearly this is impossible. Thus the supposition that $N_{x}$ and $N_{y}$ have a point in common leads to a contradiction. Now since $H$ is uncountable, the collection of 
continua $\left[N_{x}\right]$ must be uncountable. But no two of these continua have a common point, and each of them contains at least one cut point of $M$. This is contradictory to Theorem 5. Thus the supposition that Theorem 6 is not true leads to a contradiction, and the theorem is proved.

\section{THE ORDER AND REgUlarity OF THE CUT POINTS OF A CONTINUUM}

Definitions. The point $P$ of a continuum $M$ is said to be a point of Menger order $n$ of $M^{*}$ provided that for each positive number $\epsilon$ there exists a domain $U$ containing $P$ and of diameter less than $\epsilon$ and whose boundary has not more than $n$ points in common with $M$, and furthermore $n$ is the smallest positive integer such that this property is preserved. A point $P$ of a continuum $M$ will be called a regular or an irregular point of $M$ according as $M$ is or is not connected im kleinen at $P$.

THEOREM 7. Let $G$ denote the set of all the cut points of any continuum $M$. Then all save possibly a countable number of the points of $G$ are points of Menger order two of $M$.

Suppose, on the contrary, that there exists an uncountable subset $H$ of $G$ no point of which is a point of order two of $M$. Then by Theorem 3, there exist two points $A$ and $B$ of $H$ which are separated in $M$ by each point of an uncountable subset $D$ of $H$. It follows by Theorem 6 that there exists at least one point $P$ of $D$ such that every subcontinuum of $M$ which contains $P$ must contain at least one point of $D$ distinct from $P$. It is easy to see that each of the sets $M_{a}(P)$ and $M_{b}(P)$ must be connected and that $P$ is a limit point of a subset $D_{1}$ of $D$ belonging to $M_{a}(P)$ and also of a subset $D_{2}$ of $D$ belonging to $M_{b}(P)$.

Let $K_{1}$ denote the continuum $M_{a}(P)+P$ and $K_{2}$ the continuum $M_{b}(P)+P$. There exists a sequence of points $X_{1}, X_{2}, X_{3}, \cdots$, belonging to $D_{1}$ and having $P$ as its sequential limit point and such that for each positive integer $n$, $X_{n}$ precedes $X_{n+1}$ on $M$ in the order from $A$ to $B$. Now let us consider the sequence of intervals $I\left(X_{1}, X_{2}\right), I\left(X_{2}, X_{3}\right), I\left(X_{3}, X_{4}\right), \cdots$, of $M$. R. G. Lubben has shown $\dagger$ that if $N$ denotes the sequential limiting set of this sequence, then $N$ exists and is closed and connected. Clearly $N$ contains the point $P$. It must consist of $P$ alone. For suppose it does not. Then $N$ is a subcontinuum of $K_{1}$ which contains $P$. It is readily seen that $N$ cannot contain any point of the set of points

* Cf. K. Menger, Grundzüge einer Theorie der Kurven, Mathematische Annalen, vol. 95 (1925), pp. 272-306.

† See an abstract of his paper Concerning limiting sets in the Bulletin of the American Mathematical Society, vol. 32 (1926), p. 14. See also Zarankiewicz, loc. cit. 


$$
L=M_{a}\left(X_{1}\right)+\sum_{i=1}^{\infty} I\left(X_{i}, X_{i+1}\right) .
$$

But $L$ is connected and $P$ is a limit point of $L$. Hence $K_{2}+L$ is connected and contains both $A$ and $B$ but contains in common with $N$ only the point $P$. Hence $N$ contains $P$ but contains no other point of $D$. This contradicts our definition of the point $P$. Thus it follows that $N$ is identical with $P$.

Now let $\epsilon$ be any positive number. Then since the sequential limiting set of the sequence of intervals $I\left(X_{1}, X_{2}\right), I\left(X_{2}, X_{3}\right), \cdots$, consists of just the point $P$, and since $P$ is the sequential limit point of the sequence of points $X_{1}, X_{2}, X_{3}, \cdots$, it is readily seen that there exists a positive integer $k$ such that if $I$ denotes the set of points $\sum_{i=k-1}^{\infty} I\left(X_{i}, X_{i+1}\right)$, every point of $I$ is at a distance less than $\epsilon / 4$ from $P$. Now by Theorem $1, I\left(X_{k}, P\right)$ is connected, and it contains $X_{k}$. It does not contain $X_{k-1}$, because $X_{k-1}$ precedes $X_{k}$ on $M$ in the order from $A$ to $B$. Hence $I\left(X_{k}, P\right)$ must be a subset of $M_{b}\left(X_{k-1}\right)$, and since it is a subset also of $K_{1}$, it must be a subset of $I+P$. But $I+P$ is of diameter less than $\epsilon / 2$. Hence $I\left(X_{k}, P\right)$ is of diameter less than $\epsilon / 2$. In an entirely similar manner it is shown that $D_{2}$ contains a point $Y_{k}$ such that $I\left(P, Y_{k}\right)$ is of diameter less than $\epsilon / 2$. Then $I\left(X_{k}, Y_{k}\right)$ contains $P$ and is of diameter less than $\epsilon$. And since the maximal connected subset of $S\left(X_{k}, Y_{k}\right)$ which contains $P$ must have both $X_{k}$ and $Y_{k}$ as limit points, it is easily seen that there exists a domain of diameter less than $\epsilon$ which contains $P$ and whose boundary has in common with $M$ just the points $X_{k}$ and $Y_{k}$. Hence $P$ is a point of Menger order two of $M$. But by supposition $P$ belongs to $H$, and no point of $H$ is a point of Menger order two of $M$. Thus the supposition that Theorem 7 is not true leads to a contradiction.

Theorem 8. If $A$ and $B$ are any two points of a continuum $M$, and $P$ is any point belonging to $K(A, B)$ and having the property that every subcontinuum of $M$ which contains $P$ contains at least one point of $K(A, B)$ distinct from $P$, then $P$ is a point of Menger order two of $M$.

The argument given to prove Theorem 7 also proves Theorem 8 .

THEOREM 9. If $P$ is any cut point of a continuum $M$ which is a point of $M$ enger order two of $M$, then $M-P=M_{1}(P)+M_{2}(P)$, where $M_{1}(P)$ and $M_{2}(P)$ are connected point sets and $P$ is a point of order one (i.e., an end point in the Menger sense) of each of the continua $M_{1}(P)+P$ and $M_{2}(P)+P$.

TheOREM 10. If $P$ is any cut point of a bounded continuum $M$ which is a point of Menger order two of $M$, then $P$ belongs to the boundary of just one complementary domain of $M$. 
By a theorem of R. L. Moore's*, $P$ belongs to the boundary of at least one complementary domain of $M$. Suppose, contrary to this theorem, that $P$ belongs to the boundary of two complementary domains $R_{1}$ and $R_{2}$ of $M$. Then there exists $\dagger$ a simple closed curve $J$ which encloses $P$ and is of diameter less than half the diameter of $R_{1}$ and also less than half the diameter of $R_{\mathbf{2}}$, and which has in common with $M$ just two points $A$ and $B$. Then of the two arcs of $J$ from $A$ to $B$, one of them, say $A X B$, must belong to $R_{1}$, and the other, $A Y B$, must belong to $R_{2}$. But from Theorem 9 and a theorem of R. L. Moore's $\ddagger$ it follows that there exists a simple closed curve $C$ enclosing one of the sets $M_{1}(P)$ and $M_{2}(P)$ and not the other (where $M-P=M_{1}(P)$ $+M_{2}(P)$, as in Theorem 9), and containing in common with $M$ only the point $P$. It is easy to see that $C$ must contain at least one point $U$ of $A X B$ and at least one point $V$ on $A Y B$. Then if $t$ denotes the $\operatorname{arc}$ of $M$ from $U$ to $V$ which does not contain $P$, then $t$ contains no point of $M$. But this is impossible, because $U$ and $V$ belong to different complementary domains of $M$. Thus the supposition that Theorem 10 is not true leads to a contradiction.

ThEOREM 11. If $G$ denotes the set of all the cut points of a continuum $M$, then all save possibly a countable number of the points of $G$ are regular points of $M$.

Theorem 11 is a direct consequence of Theorem 7 and of Menger's $\S$ theorem that a continuum $M$ is connected im kleinen at each of its points which is a point of finite order of $M$.

ThEOREM 12. If $D$ denotes the collection of all the cut points $[P]$ of a continuum $M$ such that $P$ is an irregular point of some subcontinuum of $M$, then $D$ is countable.

Theorem 12 follows at once from Theorem 7 and the fact that any point $P$ of Menger order two of a continuum $M$ is a regular point of every subcontinuum of $M$ which contains $P$.

* R. L. Moore, Concerning the common boundary of two domains, Fundamenta Mathematicae, vol. 6 (1924), pp. 203-213.

† This follows readily from a theorem of R. G. Lubben's. See the abstract of his paper in the Bulletin of the American Mathematical Society, vol. 32 (1926), p. 114.

$\ddagger$ R. L. Moore, Concerning the separation of point sets by curves, Proceedings of the National Academy of Sciences, vol. 11(1925), pp. 469-476, Theorem 2. See also R. G. Lubben, loc. cit.

8 C. K. Menger, loc. cit. 


\section{Miscellaneous Results}

In this section some theorems will be stated with little or no proof given. These theorems, in general, either follow readily from the above propositions already proved or else they can be proved readily using methods similar to those employed in the above proofs.

ThEOREM 13. Let $G$ denote the set of all the cut points of any continuum $M$. Then $G$ is a subset of the sum of a countable number of bounded subcontinua of $M$ each of which is irreducible between some pair of points.

Theorem 13 can be proved with the aid of Lemma 2 to Theorem 5 and methods similar to those used in the proof of Theorem 4, together with the theorem of Janiszewski's* that every two points of a bounded continuum $\boldsymbol{M}$ lie together in a subcontinuum of $M$ which is irreducible between those two points.

THEOREM 14. The set $G$ of all the cut points of a continuous curve $M$ (bounded or not) is a subset of the sum of a countable number of simple continuous arcs which belong to $M$.

THEOREM 15. If $N$ is any subcontinuum of a continuum $M$ which is irreducible between some pair of points $A$ and $B$ of $M$, then all save possibly a countable number of the cut points of $M$ which belong to $N$ must separate $A$ and $B$ in $M$.

COROLlary. If $t$ is any simple continuous arc belonging to a continuous curve $M$, and $A$ and $B$ denote the end points of $t$, then all save possibly a countable number of the cut points of $M$ which lie on $t$ separate $A$ and $B$ in $M$.

* S. Janiszewski, Sur les continus irréductibles entre deux points, Journal de l'Ecole Polytechnique, (2), vol. 16 (1912).

The University of Texas, Austin, Texas 